${ }^{1}$ Radioterapia Oncológica, Instituto Nacional del Cáncer. Santiago, Chile. ${ }^{2}$ Residente Radioterapia Oncológica Universidad de Chile.

Trabajo no recibió financiamiento. Los autores declaran no tener conflictos de interés.

Recibido el 20 de marzo de 2019, aceptado el 8 de agosto de 2019 .

Correspondencia a: Javier Faúndez Salazar Avda. Profesor Zañartú 1010, Independencia, Santiago, Chile. javierfaundezs@gmail.com

\section{Complicaciones de la radioterapia: fenómeno de Lhermitte gatillado por calor. Caso clínico}

\author{
JAVIER FAÚNDEZ SALAZAR ${ }^{2}$, EVELYN SAN MARTÍN MEZA², \\ APOLO SALGADO FERNÁNDEZ ${ }^{1}$
}

\section{Complications of radiotherapy: Lhermitte phenomenon triggered by heat. Report of one case}

Lhermitte phenomenon is a neurological symptom described as a sensation of electric shock that radiates from the back towards the extremities, which appears when a patient flexes the neck. A transient myelopathy as a late complication of radiotherapy is associated with this symptom. It appears two to four months after treatment and disappears spontaneously. We report a 45 years old female with a neck malignant melanoma treated with surgery and adjuvant radiotherapy. She experienced the Lhermitte phenomenon that was triggered by heat. This phenomenon must be differentiated from the Uhthoff phenomenon.

(Rev Med Chile 2019; 147: 928-931)

(Key words: Melanoma; Multiple Sclerosis; Neoplasms, Radiotherapy; Spinal Cord Diseases.

\section{E} n el contexto de las toxicidades tardías secundarias a radioterapia, el fenómeno de Lhermitte se destaca por traducir una mielopatía totalmente benigna y transitoria (remite espontáneamente), pero que afecta tremendamente la calidad de vida de los pacientes que lo padecen. Se profundiza en el tema a propósito de un paciente cuyo dolor se gatilla, además de con la flexión cervical, al exponerse a fuentes de calor, algo no descrito previamente en la literatura. ¿Puede tratarse del fenómeno de Uhthoff: síntoma neurológico transitorio gatillado específicamente por calor? ¿Cómo hacer el diagnóstico diferencial?

\section{Caso clínico}

Paciente de 45 años, sin antecedentes mórbidos, consultó por un nódulo cutáneo cervical izquierdo de dieciocho meses de evolución, de crecimiento progresivo y que sangraba ante mínimos roces. Al examen físico destacaba una adenopatía cervical izquierda en el grupo II de 2 $\mathrm{cm}$. Una biopsia excisional concluyó: melanoma maligno nodular, Breslow $2 \mathrm{~mm}$, Indice de Clark 3 , ulcerado, índice mitótico $5 / \mathrm{mm}^{2}$, sin invasión linfovascular ni perineural. Márgenes quirúrgicos no descritos. El estudio de diseminación con PETCT FDG reveló una adenopatía cervical izquierda alta (grupo II) de $13 \times 15$ mm de diámetro, sin otro compromiso neoplásico.

Su caso fue evaluado en comité oncológico, decidiéndose una ampliación de bordes y disección cervical de grupos ganglionares izquierdos I, II, III y IV. La biopsia definitiva informó: cicatriz con neoplasia melanocítica residual a $2 \mathrm{~mm}$ del margen lateral y $5 \mathrm{~mm}$ del profundo. 21 linfonodos resecados, 1 con metástasis de melanoma (en grupo II) y compromiso extracapsular. Se etapificó como pT2b N1b M0, estadío IIIb (AJCC 2009).

Realizó radioterapia adyuvante, 46 Gy en 23 fracciones a linfonodos regionales bilaterales y 


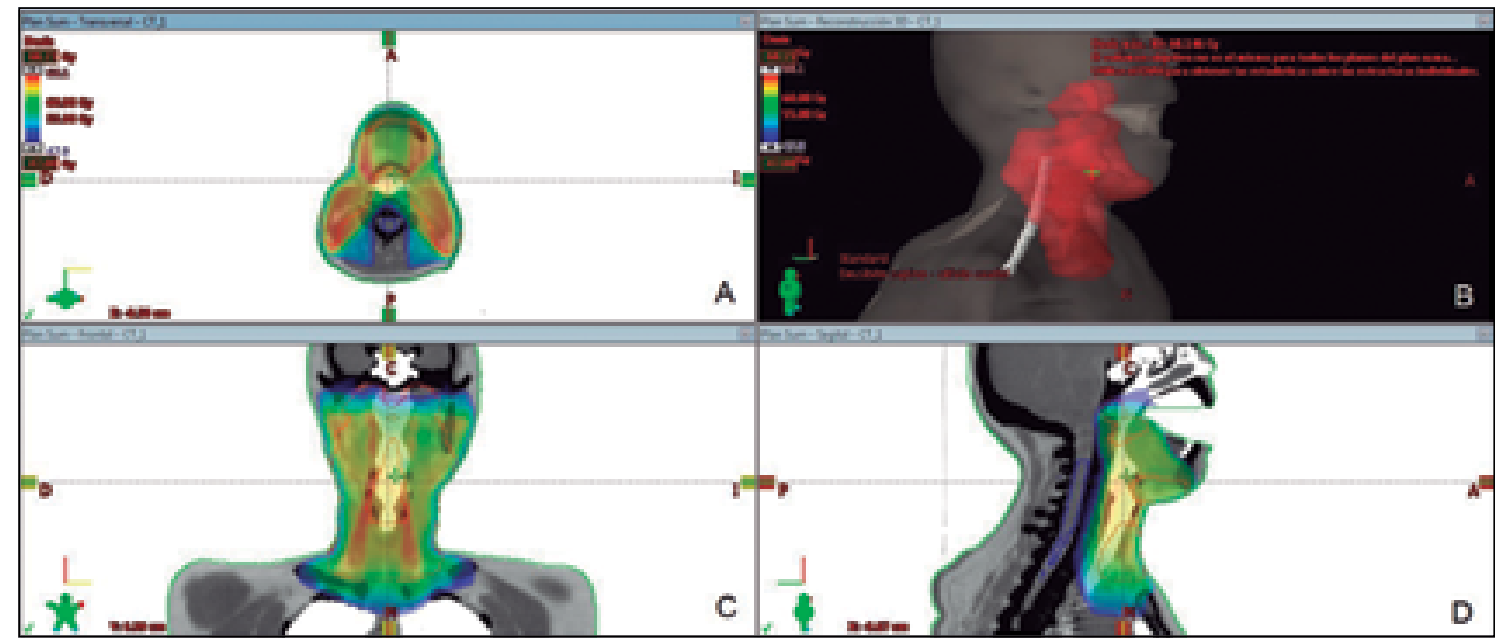

Figura 1. Dosimetría del tratamiento. A: corte transversal y representación en colores de las dosis recibidas: desde 47 Gy (azul) a 68,15 Gy (rojo). La médula espinal se marca con una línea azul; el volumen de tratamiento planificado para recibir 46 Gy (PTV1) con una línea roja, y el perímetro del cuerpo con una línea verde. B: reconstrucción 3D de la médula espinal (blanco), el PTV1 (rojo) y el cuerpo (gris). C: corte frontal. D: corte sagital. Se puede ver cómo se protege la médula espinal, la que no recibe más de 47 Gy.

lecho quirúrgico; luego, sobreimpresión a lecho hasta 60 Gy en 30 fracciones, con técnica conformada 3D, utilizando un acelerador lineal de 6 MeV. La dosis máxima en el GTV fue el 107\% de la dosis prescrita, y en médula espinal de 47 Gy (bajo el umbral de restricción de 50 Gy) (Figura 1).

Tres meses post tratamiento, el paciente presentó dolor tipo neuropático "como descargas eléctricas" en extremidades y tronco, que se desencadenaba con cambios bruscos de temperatura. $\mathrm{Al}$ interrogar sobre aquello el paciente relató que, al contacto con el agua caliente de la ducha, o al ubicarse cerca de una fuente de calor (fogata, cocina a leña) sufría el dolor ya descrito, varias veces en un mismo día. Las comidas y bebidas calientes no le provocaban dolor. No realizaba ejercicio físico intenso, por lo que no fue capaz de identificar aquello como gatillante del dolor. Al flectar el cuello, también aparecía el síntoma (aprendió a evitar aquello, con lo que disminuyó la frecuencia del dolor).

Actualmente, tras un año y diez meses luego de la radioterapia, el paciente refiere que ya no tiene dolor. Se encuentra sin evidencia de recidiva neoplásica.

El paciente dio su consentimiento para esta publicación.

\section{Discusión}

El fenómeno (o signo) de Lhermitte (FL) es un síntoma neurológico descrito como una sensación de shock eléctrico que se irradia por la espalda hacia las extremidades, que aparece cuando un paciente flecta el cuello. Entre sus causas está la irradiación de la médula espinal cervical ${ }^{1}$ cuya incidencia en series de radioterapia de cabeza y cuello ha sido reportada entre 3,6 $-13 \%{ }^{2}$. Corresponde a una mielopatía transitoria enmarcada dentro de las toxicidades tardías de la radioterapia, que aparece a los 2-4 meses después del tratamiento, y desaparece espontáneamente ${ }^{3}$.

Nuestro paciente identificó como gatillante del dolor, además de la flexión cervical, el calor. El FL causado por calor no se describe en la literatura.

$\mathrm{Al}$ indagar sobre estímulos específicos del dolor en los pacientes que sufren FL como consecuencia de radioterapia, sólo se describen los movimientos cervicales, que además de la flexión, puede ser la extensión y/o rotación ${ }^{1-4}$. Se debe tener en consideración que existen otras causas del fenómeno, y que el paciente pudiera manifestar el síntoma a raíz de alguna de ellas: esclerosis múltiple (EM) (16\% de los pacientes reportan el síntoma), quimioterapia (neurotoxicidad por cisplatino o 
docetaxel), compresión tumoral a nivel cervical de la médula espinal, trauma cervical, mielitis transversa y otras ${ }^{1}$.

Si se toma en cuenta específicamente la EM, una de sus manifestaciones se puede confundir perfectamente con lo que relató nuestra paciente: el fenómeno (signo, síndrome) de Uhthoff (FU). Éste se caracteriza por un déficit neurológico temporal, de pocos minutos de duración, desencadenado por calor. En el año 1950, el "test de la ducha caliente" era usado como herramienta diagnóstica de esta patología, en la que $60-80 \%$ de los pacientes manifiestan el síntoma ${ }^{5}$.

Entonces, cabe preguntarse ¿Cómo hacer el diagnóstico diferencial entre ambos fenómenos? La clave está en la anamnesis.

El FU es una manifestación exclusivamente de la EM. Aparece con el aumento de la temperatura corporal, tanto por ejercicio, una ducha con agua caliente, exposición al sol, ciclo menstrual, comidas o bebidas calientes y fiebre. Se manifiesta con déficit neurológicos variables dependiendo de la localización de la desmielinización, incluyendo ambliopía, ptosis, nistagmus, desincronización de los movimientos oculares binoculares, paresia, y alteración de los reflejos osteotendíneos. Se resuelve en un período de algunos minutos o hasta una hora, siempre que baje la temperatura corporal ${ }^{5}$.

Por tanto, pensar en FU implica necesariamente sospechar el diagnóstico de EM. En este caso clínico, el paciente no relató déficit neurológico alguno durante todo el tiempo que se mantuvo en control, ni tampoco tiene antecedentes mórbidos de patologías autoinmunes ni familiares con EM, todo lo cual lleva a descartar la sospecha de FU.

En cambio, el FL tiene varias causas ya mencionadas, aparece con los movimientos cervicales y se manifiesta con un dolor de carácter paroxístico. Esto último, a excepción del gatillante, se ajusta más a la historia que relató el paciente. Además, el hecho de que sus síntomas aparecieran luego de 3 meses de haber sido tratado con radioterapia de cabeza y cuello, y que con los meses remitieran espontáneamente, eleva la probabilidad diagnóstica hacia el FL.

¿Cómo se podría explicar este caso de FL gatillado por calor y por movimientos cervicales?

En relación al FU, un concepto importante es el "factor de seguridad" axonal, que se define como la relación entre la corriente del impulso y la corriente del umbral. En consecuencia, para que la conducción a través del nodo sea exitosa esta relación debe ser mayor a uno. Las fibras mielinizadas normales tienen un factor de seguridad de cinco o más. Si hay una disrupción de la mielina, la corriente se disipa a través del internodo adyacente. Por otra parte, se disminuye la cantidad de corriente disponible para despolarizar el siguiente nodo, prolongándose el tiempo de conducción internodal. Lo anterior explica la disminución en la velocidad de conducción en las fibras nerviosas afectadas por la desmielinización. Si el compromiso de la mielina progresa la corriente se torna insuficiente para despolarizar el nodo y permitir que alcance su umbral. Además, su factor de seguridad cae por debajo de uno produciéndose un bloqueo en la conducción ${ }^{5}$.

Tanto la desmielinización como el aumento de la temperatura (incluso tan poco como $0,5^{\circ}$ C) disminuyen el factor de seguridad ${ }^{5}$. La suma de ambos factores se conjuga en la manifestación clínica del FU.

Si extrapolamos lo último a nuestro caso clínico, es poco probable que estemos frente a una disminución de la velocidad de conducción o su bloqueo, pues ello no explica la generación de descargas paroxísticas de dolor. Se tiene entonces otro fundamento para no sospechar FU.

Por otra parte, el FL ocurre porque la radioterapia inhibe la proliferación de oligodendrocitos en las neuronas sensitivas ascendentes de las columnas dorsales de la médula espinal cervical y torácica superior (T1-3), lo que lleva a una desmielinización reversible. Estos axones desmielinizados adquieren características en sus canales de sodio que se asemejan a las de los mecanorreceptores y son capaces de generar descargas ectópicas en respuesta a la distorsión mecánica ${ }^{6}$ secundaria a movimientos cervicales, causando el dolor característico. Una vez que se recupera la proliferación de oligodendrocitos y la vaina de mielina vuelve a la normalidad, el FL remite. La manera específica en que los canales iónicos cambian su estructura se desconoce ${ }^{2}$.

Como hipótesis, es posible que en el paciente haya ocurrido una alteración en la estructura de los canales de sodio, que conlleve la capacidad de activar potenciales de acción ante estímulos tanto mecánicos como térmicos.

En términos prácticos, es importante que el radioterapeuta tenga un alto índice de sospecha en pacientes susceptibles de padecer FL, dado que 
es fundamental tranquilizar al paciente, el que acudirá a la consulta preocupado por la posibilidad de una progresión de su neoplasia primaria que comprometa el sistema nervioso central. Si el dolor es severo, puede haber un beneficio con carbamazepina o gabapentina ${ }^{1}$.

\section{Referencias}

1. Teoli D, Ghassemzadeh S. Lhermitte Sign. Treasure Island (FL): StatPearls Publishing. Disponible en: https:// www.ncbi.nlm.nih.gov/books/NBK493237/ [consultado el 11 de marzo de 2019].
2. Pak D, Vineberg K, Feng F, Ten Haken R, Eisbrush A. Lhermitte's syndrome after chemo-IMRT of head and neck cancer: incidence, doses, and potential mechanisms. Int J Radiat Oncol Biol Phys 2012; 83 (5): 1528-33.

3. Besa P. Radioterapia externa: lo que el médico general debe saber. Rev Med Clin Condes 2013; 24 (4): 705-15.

4. Khare S, Seth D. Lhermitte's Sign: The Current Status. Ann Indian Acad Neurol 2015; 18 (2): 154-6.

5. Panginikkod S, Rukmangadachar LA. Uhthoff Phenomenon. Treasure Island (FL): StatPearls Publishing. Disponible en: https://www.ncbi.nlm.nih.gov/books/ NBK470244/ [consultado el 11 de marzo de 2019].

6. Sá M. Physiophatology of symptoms and signs in multiple sclerosis. Arq. Neoropsiquiatr 2012; 70 (9): 733-40. 